\title{
Treatment monitoring of antiretroviral drug: on the basis of the adherence to the current antiretroviral therapy available in Bangladesh
}

\author{
[ Fariha Hossain, Dr. Raushanara Akter ]
}

\begin{abstract}
HIV is still less than $0.1 \%$ in Bangladesh, the number of newly infected patient is rising day by day. After the invention of combine ARV (antiretroviral) regiments, the success rate of antiretroviral drug has been increased dramatically. Therefore, the study has been designed to find out the factors related to adherence to antiretroviral therapy (ART). This study was designed as a cross-sectional study which was conducted through a selfdesigned questionnaire and non-probability purposive sampling technique had been used to select the sample size. The data had been collected from159 eligible HIV infected adults who have been receiving ARV for at least one year and the study had been conducted in the Hospitals of Dhaka city. This study found the current prescribed ARV regiments along with the socio-demographic characteristics of the patients and the major factors that influence the adherence rate among the patients. The findings of the study will help to strengthen the HIV/AIDS program in Bangladesh among the infected patient through proper monitoring of the treatment and also through enrich the education and proper knowledge by counseling.
\end{abstract}

Keywords-Antiretroviral drugs, HIV, AIDS, ART, ARVs

\section{Introduction}

Human Immunodeficiency virus (HIV) is considered as one of the widespread dangerous infectious killers that can lead to acquired immunodeficiency syndrome (AIDS) or even cause death if proper treatment is not given to the infected patient. HIV works by attacking the body's immune mechanism, specially the CD4 cells (T cells) which help in the maintenance of the body's immunity by fighting against any infections. If the person is being untreated then HIV reduces the significant number of CD4 cells and the immunity system become so weak that the person becomes more prone to any infections or infection related cancers.

Fariha Hossain

BRAC University

Bangladesh

Dr. Raushanara Akter

BRAC University

Bangladesh
Though there is no such treatment that can cure completely, but proper medical care can control HIV. The medicines that use to treat HIV is called antiretroviral therapy (ART) or also referred as ARV (antiretroviral) drug. Here adherence to the antiretroviral therapy (ART) or ARV (antiretroviral) medicine plays a dynamic role to suppress the virus and improve the immunity along with reducing the risk of growing drug resistance, improving the health and quality of life as well as increase the survival of the infected one.

In Bangladesh, people living with HIV get their treatment, counseling and care as free of any charge which program is led by the government of Bangladesh and supported by UNAIDS, WHO, UNICEF and UNFPA. The estimated number of people living with HIV in the country is around 11,000 (range 4,000 to 97,000) as per spectrum estimates and so far a total of 5000 persons have been diagnosed with HIV of whom 1299 are AIDS cases and 472 have died (declared by government of Bangladesh on World AIDS Day, 2017). It has been observed that, wide variations of factors are associated with influencing ART adherence among patients infected with HIV. The objectives of the present study were to identify the knowledge of the prescribed treatment to the patient, rate of adherence as well as identification of the factors related to adherence.

\section{Review of Related Literature}

A study found that the patients who have strong association with ART center through counseling, education, training and information, have the potentiality to overcome the barriers of poor adherence which can lead to the development of ARV resistant HIV (Potchoo et al,. 2010). Several researchers had been concentrated in factors associated with non-adherence, for example literacy, social support, emotional distress, unawareness etc. (Kalichman, Ramachandran and Catz, 1999). One of the study was focused on the personal barriers to non-adherence to antiretroviral drug, for example lack of family support, health and sexual life concerns, desire to have children and family instability (Mayanja et al., 2013). According to research study, knowledge and perception, childhood experience, interpersonal experience, daily environment and self-help skills are factors associated with ART adherence (Xu, Munir, Kanabkaew \& Le Coeur, 2017) 


\section{Methodology of the Study}

The study was a cross-sectional study which was carried out in Bangabandhu Sheikh Mujib Medical University (BSMMU) and Infectious Disease Hospital (IDH), these are the only HIV/AIDS health care center where free ARV are given. Both primary and secondary data had been collected throughout the survey. As the total number of the patient are not accessible, therefore 159 eligible HIV infected adults were interviewed who have been receiving ARV for at least one year. 35 sets of questions were asked the patients and the enrolment was voluntarily because of ethical issues.

\section{Iv. Data analysis}

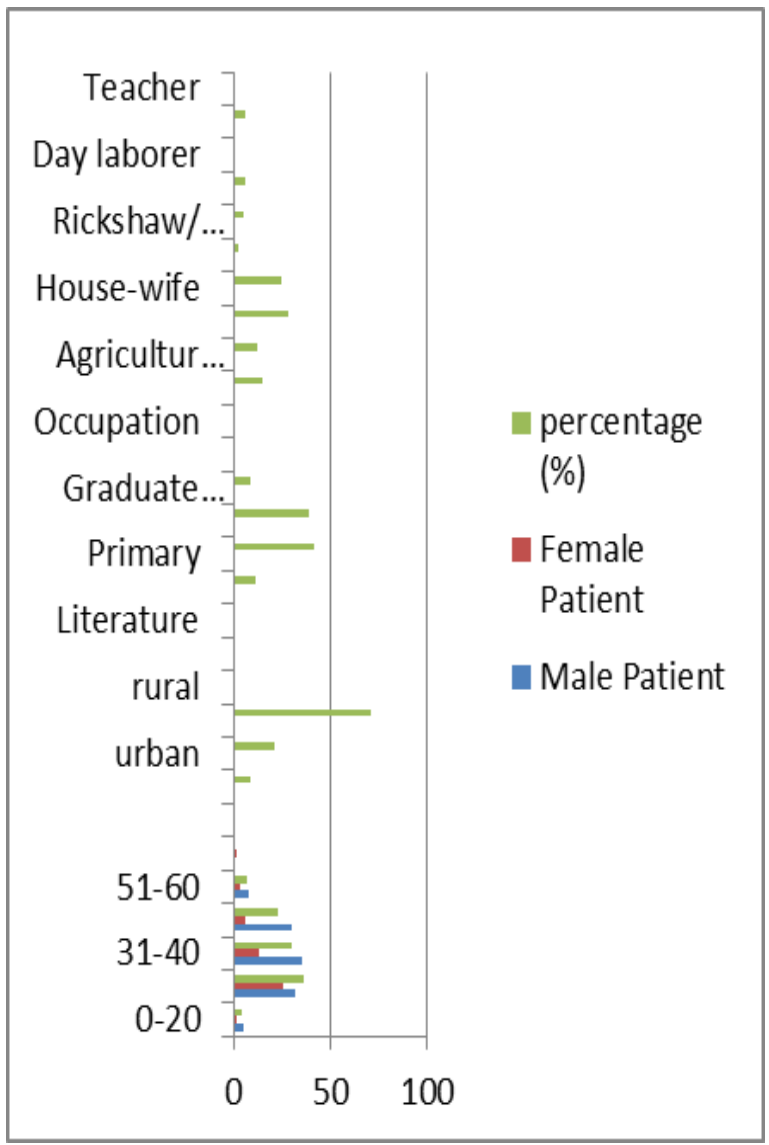

Figure 1. The socio-demographic characteristics of the HIV infected patient

Figure 1 represents the socio demographic characteristics of the total number of sample $(n=159) .69 .18 \%$ were male patients and $30.82 \%$ were female patients. The age of the patients were ranged from 5-70 years. $71.07 \%$ of total patient were from rural area of Bangladesh along with $8.18 \%$ and $20.75 \%$ were from urban and semi urban are respectively. $88.68 \%$ of the patient were educated and among all the patient $47.17 \%$ patients had been able to say the name of their prescribed medicines and they had been found aware about the misuse or improper use of the ARV.

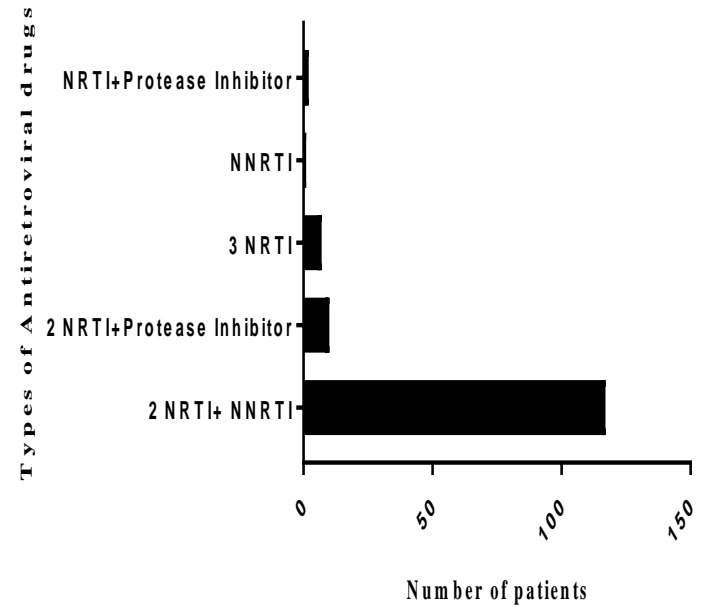

Figure 2. Distribution of the HIV infected patient $(n=159)$ according to the prescribed ARVs combination

Figure 2 represents that most of the patients have been receiving combination of three ARVs $(84.28 \%$ of the patient). $0.63 \%$ has been receiving Non-Nucleoside Reverse Transcriptase Inhibitors (NNRTI) and $1.26 \%$ has been receiving Nucleoside Reverse Transcriptase Inhibitors (NRTI) +Protease Inhibitor combination. $74.58 \%$ of the total patients have been receiving 2 Nucleoside Reverse Transcriptase Inhibitors (NRTI) + Non-Nucleoside Reverse Transcriptase Inhibitors (NNRTI), 6.29\% have been receiving 2 Reverse Transcriptase Inhibitors (NRTI) +Protease Inhibitor and $4.40 \%$ of them have been receiving 3 Nucleoside Reverse Transcriptase Inhibitors (NRTI).

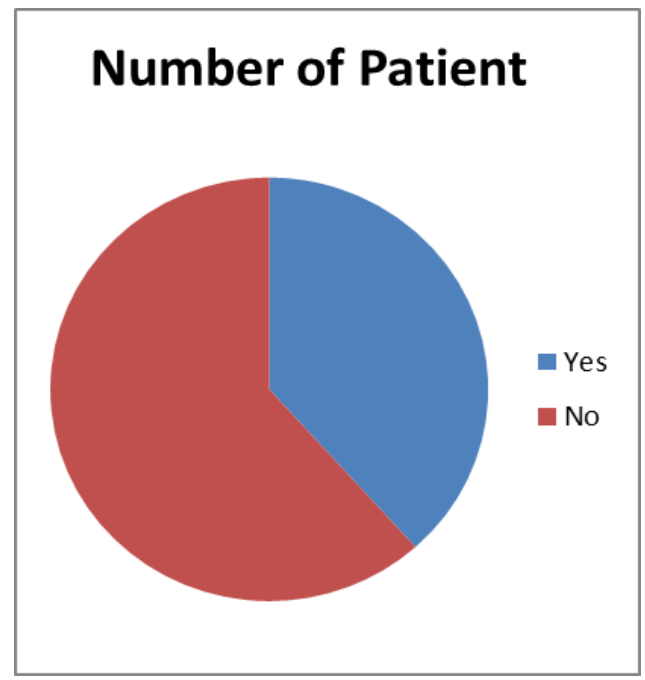

Figure 3. percentage of the patient according to the missed dose in last seven days before the interview was hold.

Figure 3 When the patients were asked about the missing doses of last seven days, then $38.36 \%$ of the patient had been replied affirmatively and $61.64 \%$ of the patient had been replied negatively. 


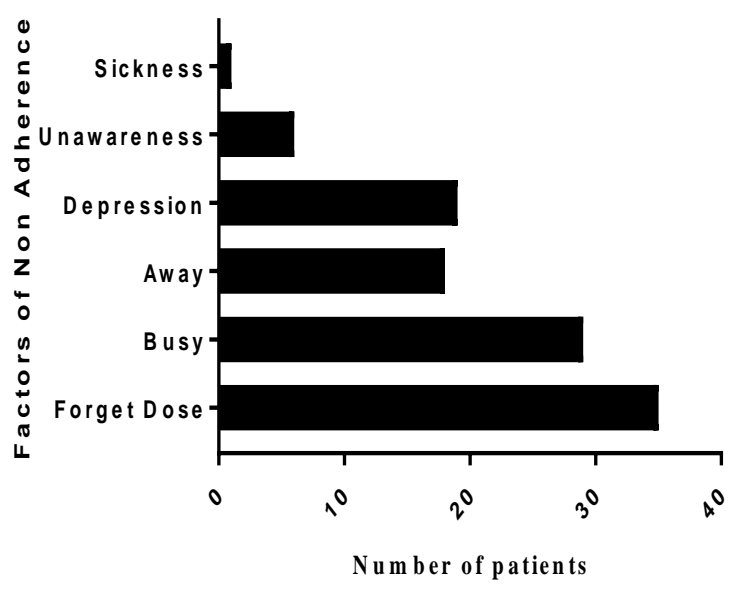

Figure 4. Distribution of the HIV infected patient $(n=159)$ according to the factors of non-adherence.

Figure 4 represents the factors that influence the adherence rate of the patients. $22.01 \%$ patients had reported that they just forgot to take the medication and it was found in similar studies. Forgetting to take the medicine had been identified as the first main barrier to adherence. Then, $18.24 \%$ of the patient had reported being busy as their barrier to adherence and this had been found as the second main reason of poor adherence. Away from home and forget to travel with the medicine had been the third main reason of non-adherence. Depression had been identified as a major reason behind the non-adherence, because the patients were subjected to the social stigma and discrimination. This psychological status led to neglect toward having the medicines in time. Unawareness (3.77) and sickness (0.63) were found as the minor reason to non-adherence, because the patients had been counseling by medical personnel for several times. So that, most of the patient knew about the demerits of not taking medicines and whenever they felt any sickness physician or counselor were available via telecommunication.

\section{v. Findings of Study}

The study had been concentrated on the identification of the factors influencing the adherence rate among the patients. Throughout the interview session it has been found out that the self-reported assessment of adherence level through interview would be an acceptable, simple and easiest method in Bangladesh and this monitoring would have encourage the patient to strictly adhere to the prescribed medicines. However, this study did not find any significant associations with the socio-demographic characteristics accept literacy. The major factors, which are forgetting, being busy, away from home and depression are mostly related to personal barrier among the patients. Other minor factors such as sickness and unawareness are likely less happened.

\section{vI. Conclusions}

In conclusion it can be said that, the present scenario of Bangladesh has showed that its HIV prevalence is still lower than any other countries of Asia. However, this study has shown the quality of the given treatment along with the knowledge and capacity to adhere to ART of the infected patient. Since ART is a lifelong treatment, therefore HIV infected patient have to be well monitored in a regular basis by trained medical professionals to help the patient to overcome the barriers that they have been facing throughout their treatment.

\section{References}

[1] Mayanja, B., Kabunga, E., Masiira, B., Lubega, R., Kaleebu, P., \& Seeley, J. (2013). Personal barriers to antiretroviral therapy adherence: Case studies from a rural Uganda prospective clinical cohort. African Health Sciences, 13(2)

[2] Kalichman, S., Ramachandran, B., \& Catz, S. (1999). Adherence to combination antiretroviral therapies in HIV patients of low health literacy. Journal Of General Internal Medicine, 14(5), 267-273.

[3] Xu, L., Munir, K., Kanabkaew, C., \& Le Coeur, S. (2017). Factors influencing antiretroviral treatment suboptimal adherence among perinatally HIV-infected adolescents in Thailand. PLOS ONE, 12(2), e0172392.

[4] A PROSPECTIVE STUDY OF PREDICTORS OF ADHERENCE TO COMBINATION ANTIRETROVIRAL MEDICATION. (2002). Infectious Diseases In Clinical Practice, 11(5), 325.

[5] WHO|Antiretroviral therapy. (2017). Who.int. Retrieved 26 December 2017, from http://www.who.int/topics/antiretroviral_therapy

[6] Islam, M., Mitra, A., Mian, A., \& Vermund, S. (1999). HIV/AIDS in Bangladesh: a national surveillance. International Journal Of STD \& AIDS, 10(7), 471-474.

[7] Odeny, T., Penner, J., Lewis-Kulzer, J., Leslie, H., Shade, S., \& Adero, W. et al. (2013). Integration of HIV Care with Primary Health Care Services: Effect on Patient Satisfaction and Stigma in Rural Kenya. AIDS Research And Treatment, 2013, 1-10.

[8] Oguntibeju, O. (2012). Quality of life of people living with HIV and AIDS and antiretroviral therapy. HIV/AIDS - Research And Palliative Care, 117.

[9] Johnson, M., Dilworth, S., Taylor, J., Darbes, L., Comfort, M., \& Neilands, T. (2011). Primary Relationships, HIV Treatment Adherence, and Virologic Control. AIDS And Behavior, 16(6), 15111521.

[10] Hornschuh, S., Dietrich, J., Tshabalala, C., \& Laher, F. (2017). Antiretroviral Treatment Adherence: Knowledge and Experiences among Adolescents and Young Adults in Soweto, South Africa. AIDS Research And Treatment, 2017, 1-8.

[11] Guidelines for managing advanced HIV disease and rapid initiation of antiretroviral therapy. (2017). World Health Organization. Retrieved 26 December 2017, from http://bit.ly/2pE5JS8

About Author (s):

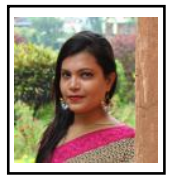

Fariha Hossain is studying Bachelor in Pharmacy IN BRAC University and will be graduated by January, 2018. Her preferred research areas include Pharmacoloy, Clincal Pharmacy, Medicine uses and safety.

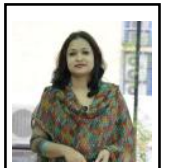

Dr. Raushanara Akter joined the Department of Pharmacy at BRAC University as an Assistant Professor in July, 2015. She started her professional career as a Product Development Officer at Beximco Pharmaceuticals Ltd. 